\title{
Consumo de bebidas en pré-escolares
}

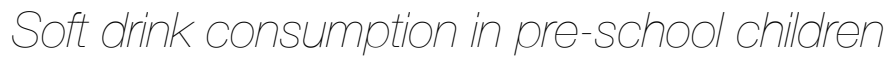

Pistochini, A

Pisaniak, $S$

Marco, $V$

Doño, $R$

Introducción: Los hogares han incorporado masivamente el consumo de bebidas ácidas. El propósito del presente estudio fue determinar en alumnos de un jardín de infantes el patrón de consumo de bebidas de uso frecuente y el estado dentario.

Material y métodos: Sobre un total de 53 alumnos de una jardín público, 21 hombres y 32 mujeres con una edad promedio de 3,86( $\pm 0.98 \mathrm{DS})$, se realizó una encuesta a los padres de carácter anónimo, referida a salud general, medidas preventivas educativas y consumo de bebidas. Sobre una muestra de 26 alumnos seleccionados aleatoriamente se realizó examen dentario y se confeccionó ceod, ceos, pérdida de tejido dentario, según Smith y Knight (1984). Se realizó distribución de la frecuencia de las variables de la encuesta; media y ES de los indicadores dentarios y correlación de las variables.

Resultados: La distribución de frecuencia mostró: recibe medicamentos 9\%, presenta antecedentes de alergia $28 \%$, tiene asma $6 \%$. Los momentos de azúcar $x$ fue de 4,76 $\pm 0,219$ El 19\% recibe aplicación tópica de fluoruros, $73 \%$ usa pastas fluoradas. El $41 \%$ se cepilla 2 veces por día; .Las bebidas preferidas resultaron los jugos, $89 \%$. El 69\% gaseosas, $28 \%$ bebidas deportivas y un $19 \%$ bebidas a base de soja. El consumo antes de dormir era de un $40 \%$,y el $11 \%$ usaba ma- madera. El ceod fue de $3.38 \pm 0,779$, con un componente cd de 2,961 $\pm 0,665$ y el ceos de $5 \pm 1,708$, con un cs de 3,84 $\pm 1,05$. El 81\% presentó pérdida de tejido dentario, principalmente o/y Bucal.

Conclusión: La escasa aplicación de medidas preventivas, los patrones de consumo de bebidas en edades tempranas y el incremento de las erosiones hace necesario implementar acciones de educación para la salud en las escuelas.

Palabras clave: hábitos de consumo, preescolares, bebidas ácidas, erosión.

\section{Abstract \\ Consumption of acidic beverages has become commonplace in the average home. The aim of the present study was to evaluate beverage con- sumption habits and dental status in preschool children of a kindergarten.}

The study population comprised 53 children attending a state kindergarten in Buenos $\mathrm{Ai}$ res city, including 21 boys and 32 girls; average age was 3.86 years ( \pm SD 0.98 ). The parents of the children filled in a questionnaire inquiring about general health, educational preventive measures, and beverage consumption. Twenty six children were randomly selected and subjected to dental examination, determination of 
their dmft and dmfs scores, loss of dental tissue according to Smith and Knight (1984). Frequency distribution of the variables included in the questionnaire was determined, mean and SE of the dental and salivary indicators was calculated, and correlation among variables was analyzed.

Frequency distribution showed that 9\% took medication, 28\% suffered from allergies, $6 \%$ suffered from asthma. The mean of sugar consumption was $4,76 \pm 0,219 \mathrm{SE}$. Nineteen percent received topical applications of fluoride, 73\% used fluoridated toothpaste. Forty one percent brushed their teeth 2 times a day.

The prefer beverage were regular juices in $89 \%$ of cases; $69 \%$ soft drinks, $28 \%$ sport drinks, $19 \%$ drank soja.

The $40 \%$ consumpted before go to bed and eleven percent taked botle $\mathrm{dmft}$ was $3.38 \pm 0,779$ (SE) the dt was 2,961 $\pm 0,665$ (SE)and dmfs was $5 \pm 1,708$ (SE). The ds 2,961 $\pm 0,665$ (SE) eighty one percent of clhildren presented loss of dental tissue; presented tissue loss on the occlusal / incisal and buccal surface.

The lack of preventive measures, the observed beverage consumption habits in early ages, and the increase in dental erosion, evidence the need to implement health education programs in schools.

Key words: consumption habits, preschool children, acidic beverages, erosion.

\section{Introducción}

La erosión dental es la pérdida patológica, irreversible, de tejidos duros de la superficie dentaria que responde a un proceso químico, no bacteriano (Pindborg 1970) ${ }^{1}$ En las últimas dé- cadas ha marcado un incremento clínicamente significativo (Milosevic et al 1997). ${ }^{2}$

La erosión dental puede tener una etiología multifactorial debida a causas extrínsecas o intrínsecas. Los ácidos intrínsecos (gástricos, de regurgitación o vómitos) y extrínsecos (dieta) han sido relacionados como el principal factor etiológico, iniciando los procesos químicos que conducen a la disolución mineral de inicio, al reblandecimiento y consecuentemente a la pérdida de la superficie de tejido dentario. (Zero 2005) $)^{3}$

Los factores extrínsecos involucran la conducta alimentaria, medicaciones y estilo de vida.

Un informe realizado por el Instituto Nacional de Salud de los Estados Unidos (NIH) en el año 1997, aseguraba que en las últimas tres décadas, había bajado el consumo de leche, había disminuido también la dieta que contenía calcio tanto en los niños como en los adultos ya que sustituían su ingesta por gaseosas y jugos artificiales.

Según Sohn W, Burt BA (2006) ${ }^{4}$ hay suficiente evidencia que soporta que los niños en Estados Unidos han aumentado la diversidad en el consumo de bebidas, reemplazando la leche y el agua por bebidas carbonatadas y jugos de frutas. La relación entre bebidas carbonatadas y caries mostró informes contradictorios.

En los últimos años se ha observado un incremento en el consumo de las bebidas erosivas. Diferentes autores han relacionado el hábito de consumo de bebidas con pérdida de tejido dentario de origen no bacteriano (Shellis 2005) ${ }^{5}$.

Está incrementando la prevalencia de erosión (Armadottir 2003, Nunn 2003) (-7 $^{6}$ el consumo de gaseosas está siendo reconocido como uno 
de los factores principales (Johansson 1997) ${ }^{8}$. Estudios clínicos han mostrado que el consumo de bebidas no alcohólicas, entre las cuales se incluyen jugos y gaseosas, están involucradas debido a su bajo pH (Jendottir 2004) ${ }^{9}$.

En Venezuela del Rea (2005) ${ }^{10}$ encontró que la leche se encuentra dentro de los 12 alimentos más consumidos por los preescolares, siendo en un $74 \%$ los preescolares y un $63 \%$ los escolares.

Según Nielsenn Company (2008) en Argentina los jóvenes de 12 a 19 años consumían el 85\% bebidas gaseosas.

El propósito del presente estudio fue determinar en alumnos de un jardín de infantes el patrón de consumo de bebidas de uso frecuente y el estado dentario.

\section{Material y métodos}

Sobre un total de 53 alumnos de un jardín público de la Ciudad Autónoma de Buenos Aires, 21 hombres y 32 mujeres con una edad promedio de 3,86 ( $\pm 0.98 \mathrm{DS})$, se realizó un cuestionario de carácter anónimo a los padres, referido a salud general, medidas preventivas educativas y consumo de bebidas. 24 preguntas, 3 de datos personales (edad, sexo, trabajo de los padres), 5 de salud general, 11 de antecedentes odontológicos, 5 de patrones de consumo en relación a veintiuna bebidas de consumo frecuente.

Sobre una muestra de 26 alumnos seleccionados aleatoriamente se realizó examen dentario y se confeccionó ceod, ceos, pérdida de tejido dentario, según Smith y Knight (1984) ${ }^{11}$. Se realizó distribución de la frecuencia de las variables de la encuesta; media y ES de los indicadores dentarios y correlación de las variables.

\section{Análisis estadístico}

Las respuestas obtenidas fueron volcadas a planillas Excel, calculando la distribución de la frecuencia de las variables, la media, la desviación estándar y el error estándar. Se analizaron las asociaciones y correlaciones de las variables con el estado dentario por chi cuadrado (X2) y coeficiente de correlación de Spearman.

\section{Resultados}

El 60\% de la población eran niños y el $40 \%$ niñas, con una edad promedio de 3,86 ( \pm 0.98DS). (MINIMA 3 MAXIMA 5).

El $72 \%$ trabajaban los padres, el 9\% recibía medicación, un $28 \%$ manifestó tener alergia, y un $6 \%$ asma, ninguno presentaba regurgitación (Cuadro 1).

Cuadro 1. N: 53 - edad promedio: 3,86 ( \pm 0.98DS)

$72 \%$ trabaja el padre

\begin{tabular}{|c|c|}
\hline Recibe medicamentos & $9 \%$ \\
\hline Alergia & $28 \%$ \\
\hline Asma & $6 \%$ \\
\hline
\end{tabular}

Se registró un promedio de $4.76 \pm 0.219$ (ES) de momentos de azúcar.

Solo un $19 \%$ recibe aplicación profesional de fluoruros tópicos.

El 73\% utiliza pastas fluoradas. Un $4 \%$ realiza buches fluorados. (Cuadro 2).

El $41 \%$ dice realizar 3 cepillados por día y un $17 \%$ dos, El 76\% desconoce la técnica y el $58 \%$ la realiza con cepillo suave. (Cuadro 3 ). 
Cuadro 2. Momentos de azúcar: $4.76 \pm 0.21$

\begin{tabular}{|c|c|}
\hline Recibe topicación & $19 \%$ \\
\hline Utiliza dentífricos & $73 \%$ \\
\hline Realiza enjuagatorios & $4 \%$ \\
\hline
\end{tabular}

Cuadro 3. Cepillado diario:

$17 \% 2$ veces por día

$41 \% 3$ veces por día

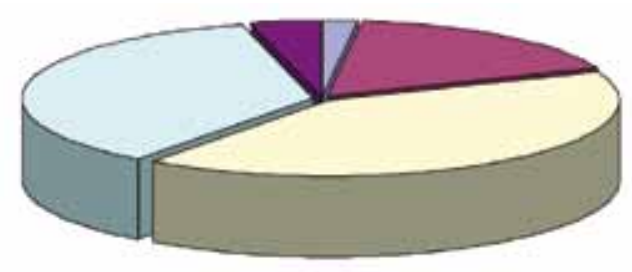

El $76 \%$ desconoce la técnica

Técnica de cepillado

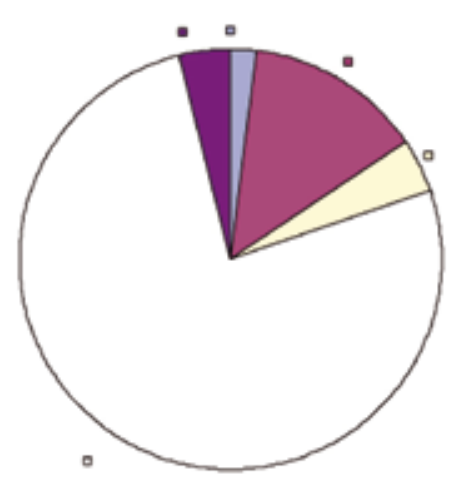

$58 \%$ con cepillo suave

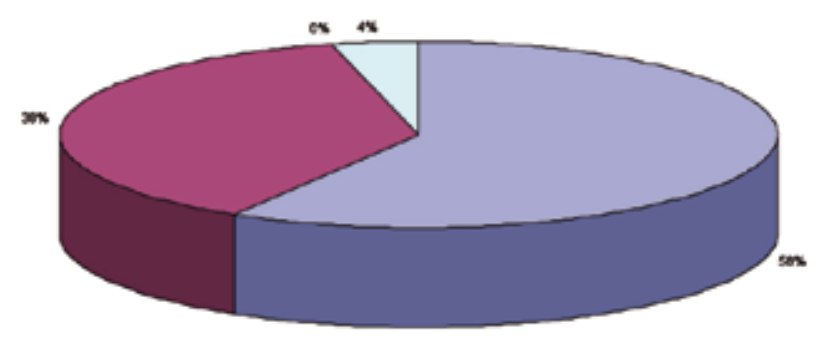

El cuestionario de consumo de bebidas reveló:

El 94\% marca como bebida preferida la leche, $51 \%$ leche entera y un $15 \%$ saborizadas.
El $89 \%$ prefiere el yogurt, de este un $77 \%$ la modalidad entero.

El 96\% responde el agua, y de este el $40 \%$ agua corriente y un $22 \%$ aguas saborizadas.

Las gaseosas son preferidas en un $75 \%$, de esta un $63 \%$ del modo regular.

El $89 \%$ selecciona los jugos, y de estos un $43 \%$ natural, y otro $43 \%$ en polvo diluido.

El 28\% elige las bebidas deportivas y solo un 19\% las bebidas a base de soja. (Cuadro 4).

Cuadro 4. Consumo de bebidas: bebida preferida

\begin{tabular}{|c|c|c|}
\hline Leches & $94 \%$ & $51 \%$ entera \\
\hline Yogures & $89 \%$ & $77 \%$ entero \\
\hline Aguas & $96 \%$ & $40 \%$ corriente \\
\hline Gaseosas & $75 \%$ & $63 \%$ regular \\
\hline Jugos & $89 \%$ & $\begin{array}{c}43 \% \text { jugo natural } \\
43 \% \text { polvo diluido }\end{array}$ \\
\hline Bebidas deportivas & $28 \%$ & \\
\hline Bebidas de soja & $19 \%$ & \\
\hline
\end{tabular}

$\mathrm{Al}$ analizar la frecuencia semanal registran un consumo de leche entera del $83 \%$, descremada $12 \%$ y fermentadas $9 \%$ todos los días de la semana, las saborizadas en un $11 \%$ en forma irregular.

En relación a los yogures un $37 \%$ consume la forma entera todos los días.

El agua corriente la consumen en un $54 \%$ todos los días, y la mineral sin gas un $30 \%$.

Las aguas saborizadas se consumen en forma irregular en un $10 \%$ 
Cuadro 5. Consumo semanal

\begin{tabular}{|c|c|c|}
\hline Leche entera & $83 \%$ & Todos los días \\
\hline Yogur & $37 \%$ & Todos los días \\
\hline Agua corriente & $54 \%$ & Todos los días \\
\hline Gaseosas & $26 \%$ & Todos los días \\
\hline Jugo en polvo & $20 \%$ & Todos los días \\
\hline Bebidas deportivas & $24 \%$ & Irregularmente \\
\hline Bebidas de soja & $20 \%$ & Irregularmente \\
\hline
\end{tabular}

Las gaseosas regulares en un $26 \%$ en forma irregular y un $10 \%$ todos los días.

El jugo en polvo un $20 \%$ todos los días, y 17\% en forma irregular.

El jugo exprimido en un $31 \%$ de forma irregular.

La frecuencia semanal de bebidas deportivas fue de $24 \%$ irregularmente y las bebidas a base de soja en un $20 \%$ de la misma modalidad. (Cuadro $5)$.

Al analizar la frecuencia diaria un 55\% de la población manifestó consumir leche entera 2 veces por día y leche saborizada un 13\% de forma irregular. Yogur entero un $27 \% 1$ vez por día. El $48 \%$ consume agua corriente más de 4 veces al día y agua mineral sin gas el $20 \%$ con la misma frecuencia. En relación a las gaseosas regulares un $17 \%$ las consume 2 veces por día y el $27 \%$ más de 4.

El jugo exprimido es consumido en un $20 \%$ forma irregular, el jugo envasado un $10 \%$ y el polvo diluido un $24 \% 3$ veces por día.
Cuadro 6. Frecuencia diaria

\begin{tabular}{|c|c|c|}
\hline Leche entera & $55 \%$ & 2 veces por día \\
\hline Yogur & $27 \%$ & 1 vez por día \\
\hline Agua corriente & $48 \%$ & Más de 4 veces/ día \\
\hline Gaseosas & $27 \%$ & Más de 4 veces/ día \\
\hline Jugo en polvo & $24 \%$ & 3 Veces \\
\hline Bebidas deportivas & $24 \%$ & Irregularmente \\
\hline Bebidas de soja & $13 \%$ & Irregularmente \\
\hline
\end{tabular}

Las bebidas deportivas un $24 \%$ de forma irregular y las a base de soja un $13 \%$ también en forma irregular. (Cuadro 6).

$\mathrm{Al}$ consultar el momento de consumo el $41 \%$ manifestó lo hace durante la noche y antes de dormir 9\%.

En cuanto al modo de suministrar la bebida el $11 \%$ aun utiliza mamadera, el $11 \%$ vaso o taza, $64 \%$ usa bombilla. El 19\% manifestó utilizar taza y bombilla.

Para realizar el examen clínico fueron seleccionados de forma aleatoria 26 niños. 35\% varones

Cuadro 7. Experiencia clínica

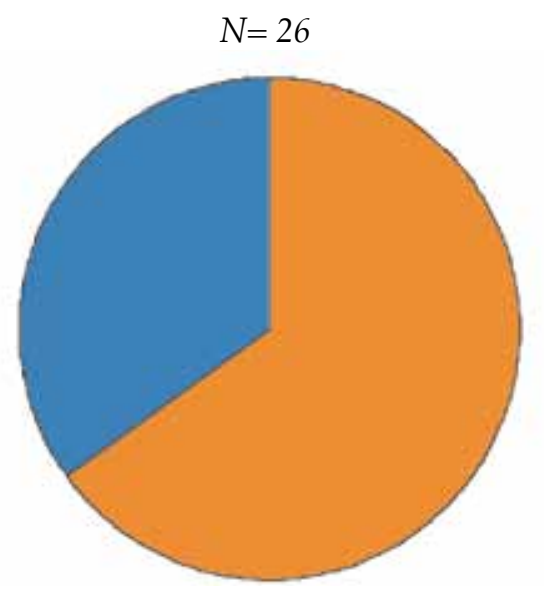


y $65 \%$ niñas, (Cuadro 7) con una edad promedio de $3,42 \pm 0,75$ (DS).

Del registro dentario se obtuvo:

Un ceod de 3.38 \pm 0.77 (DS), con los componentes cd de $2.96 \pm 0.66$ (ES);

$\mathrm{Y}$ el ceos de $5 \pm 1.70$ (ES) y un componente cs de $3.84 \pm 1.05$ (ES) (Cuadro 8).

Cuadro 8. Experiencia clínica. $N=26$

\begin{tabular}{|c|c|}
\hline Edad & $3,42 \pm 0,75(\mathrm{DS})$ \\
\hline Ceod & $3,38 \pm 0,77(\mathrm{ES})$ \\
\hline Cd & $2,96 \pm 0,66(\mathrm{ES})$ \\
\hline Ceos & $5 \pm 1,70(\mathrm{ES})$ \\
\hline Cs & $3,84 \pm 1,05(\mathrm{ES})$ \\
\hline
\end{tabular}

Cuadro 9. Experiencia clínica Pérdida dentaria: $81 \%$

\begin{tabular}{|c|c|}
\hline O/I & $X=0.25 \pm 0.06(\mathrm{ES})$ \\
\hline $\mathbf{L}$ & $\mathrm{X}=0.1 \pm 0.03(\mathrm{ES})$ \\
\hline
\end{tabular}

Se observó un $81 \%$ de pérdida dentaria, obteniendo las medias de pérdida dentaria ocluso incisal de $0.25 \pm 0.06$ y lingual de $0.1 \pm 0.03$. (Cuadro 9).

Al realizar el procesamiento estadístico se hallaron asociaciones y correlaciones entre:

- Bebida preferida jugo con pérdida dentaria ocluso incisal, chi cuadrado de 5.13 con una $\mathrm{p}<0.02$ y un rho de 0.37 con un valor de $\mathrm{p}$ $<0.05$.
- Frecuencia de consumo de de leche entera y componente cd del ceod, con un chi cuadrado de 10.71 y un $\mathrm{p}<0.001$ y un valor rho de 0.51 y $\mathrm{p}<0.01$.

- Frecuencia de consumo de agua saborizada y perdida dentaria ocluso incisal con un chi cuadrado de 4.15 y p $<0.05$ y un rho de 0.37 $\mathrm{p}<0.05$.

- Frecuencia de consumo de jugo exprimido y perdida dentaria con un chi cuadrado de 4.019 y un $p<0.05$ y un rho de $0.37, p<0.05$.

- Frecuencia de consumo de jugo liquido y perdida dentaria lingual, con un chi cuadrado de10.18 y p <0.001 y un rho de 0.43 , $\mathrm{p}<0.03$.

- Frecuencia diaria de gaseosa y perdida dentaria ocluso incisal, con un chi cuadrado de 4.36 y una $\mathrm{p}<0.04$ y un rho de $0.39<0.05$. (Cuadro 10).

\section{Discusión}

En el presente trabajo solo el 19\% de los niños recibían aplicación profesional de fluoruros tópicos y un $73 \%$ manifestó utilizar pastas fluoradas, un $17 \%$ se cepilla 2 veces diarias y un $41 \%$ tres, valores menores a los registrados por Liu (2007) 12 en una población de similares características en Beijing, China, donde el $88 \%$ de los niños utilizaba pastas y el $74 \%$ de la población realizaban 2 o más cepillados diarios.

Sin embargo, los resultados obtenidos son similares a los hallados por este grupo cuando se aplicó este cuestionario en adolescentes de escuelas secundarias $(2008)^{13}$.

Los hábitos alimentarios están influídos por una amplia gama de factores, especialmente por as- 
Cuadro 10. Experiencia clínica.

\begin{tabular}{|c|c|c|c|c|}
\hline & $\mathbf{X 2}$ & $\mathbf{P}$ & rho & P \\
\hline Bebida preferida jugo- OI & 5.13 & $<0.02$ & 0.37 & $<0.05$ \\
\hline Frecuencia leche entera-cd & 10.71 & $<0.001$ & 0.51 & $<0.01$ \\
\hline Frecuencia agua saborizada-OI & 4.15 & $<0.05$ & 0.37 & $<0.05$ \\
\hline $\begin{array}{c}\text { Frecuencia jugo exprimido-Perdida } \\
\text { dentaria }\end{array}$ & 4.019 & $<0.05$ & 0.37 & $<0.05$ \\
\hline Frecuencia jugo líquido-L & 10.18 & $<0.001$ & 0.43 & $<0.03$ \\
\hline Frecuencia diaria gaseosa-OI & 4.36 & $<0.04$ & 0.39 & $<0.05$ \\
\hline
\end{tabular}

pectos socioculturales y económicos que determinan en mayor o menor grado la disponibilidad y el acceso a los alimentos. (Nestlé M et al 1998) ${ }^{14}$.

El 94\% manifiesta elegir la leche como bebida preferida, el $55 \%$ de la población consume 2 veces por día, valores que no coinciden con las presentadas en la literatura donde se manifiesta una disminución del consumo de leche. (Sohn et al 2006 $)^{4}$ y superior a los hallados por del Rea $(2005)^{10}$ en Venezuela.

El $75 \%$ elige las gaseosas y el $89 \%$ los jugos en sus diversas formas. En estudios previos quedo demostrado el poder erosivo de estas bebidas de bajo pH y elevado efecto buffer (Argentieri 2002 $)^{15}$ hallándose correlación significativa entre los jugos y la perdida dentaria.

Los momentos de azúcar presentaron una media de 4.76. Si bien no se encontró correlación significativa con el estado dentario podrían estos ser factores de los valores obtenidos en el ceod y la correlación significativa de consumo de leche con componente cd, por el agregado de azúcar a la leche, la utilización de mamadera y / o bombilla y el consumo nocturno.

El porcentaje elevado de perdida de tejido de origen no bacteriano (81\%) es superior al 52\% presentado por Nunn J et al, en Reino Unido, del reporte 1996/977 en una población de edad similar, que a su vez demostraba un incremento de la prevalencia de erosión comparado con los registros de 1993 en el mismo país. Millward 16 et al en 1994 en esta edad registro solo un 38\%.y Deshpande et $\mathrm{al}^{17}, 2004$, solo un $28.57 \%$.

Las asociaciones y correlaciones encontradas entre gaseosas, jugos con pérdida de tejido dentario hacen necesario analizar el consumo de este tipo de bebidas en las escuelas a nivel local como lo realizado por la academia americana de pediatría donde recomienda sobre el control de este tipo de bebidas en las escuelas.

\section{Conclusiones}

- El 94\% de los preescolares eligen como bebida preferida la leche. 
- El 89\% de los preescolares eligen los jugos.

- Se encontró asociación y correlación entre pérdida dentaria y consumo de jugos y gaseosas
- La escasa aplicación de medidas preventivas, los patrones de consumo de bebidas en edades tempranas y el incremento de las erosiones hace necesario implementar acciones de educación para la salud en las escuelas.

\section{Bibliografía}

1. Pindborg JJ Pathology of dental hard tissues. Copenhagen: Munskgaard (1970):312-321

2. Milosevic A, Kelly MJ, McLean AN. Sport supplement drinks and dental health in competitive swimmers and cyclist. Br Dent J (1997); 182:303-308

3. Zero DT, Lussi A. Erosion--chemical and biological factors of importance to the dental practitioner. Int Dent J. (2005);55(4 Suppl 1):285-90.

4. Sohn W, Burt BA, Sowers MRCarbonated Soft Drinks and Dental Caries in the Primary Dentition J Dent Res (2006) 85(3):262-266

5. Shellis RP, Finke M, Eisenburger M, Parker DM, Addy M. Relationship between enamel erosion and liquid flow rate. Eur J Oral Sci. (2005) Jun;113(3):232-8.

6. Arnadottir IB, Sæmundsson SR, Holbrook WP Dental erosion in Icelandic teenagers in relation to dietary and lifestyle factors. Acta Odontol Scand (2003). 61:25-28.

7. Nunn JH, Gordon PH, Morris AJ, Pine CM, Walker A. Dental erosion—changing prevalence? A review of British National children's surveys. Int J Paed Dent (2003) 13:98-105.

8. Johansson AK, Johansson A, Birkhed D, Omar R, Baghdadi S, Khan N, Carlsson GE (1997). Dental erosion associated with soft-drink consumption in young Saudi men. Acta Odontol Scand 55:390-397.

9. Jensdottir T, Holbrook P, Nauntofte B., Buchwald C, Bardow A. Immediate Erosive Potential of Cola Drinks and Orange Juices J Dent Res ( 2006) 85(3):226-230,

10. Del Rea S, Fajardo Z, Solano L et al. Patrón de consumo de alimentos en niños de una comunidad urbana al norte de Valencia, Venezuela. ALAN, (2005), vol.55, no.3, 279-286.

11. Smith BGN. Knight JK. A comparison of patterns of tooth wear with aetiological factors. Br Dent J. (1984);157:16

12. Liu M, Zhu L, Zhang B, Petersen PE. Changing use and knowledge of fluoride toothpaste by schoolchildren, parents and schoolteachers in Beijing, China. Int Dent J. (2007) Jun;57(3):187-94.

13. Pistochini A; Argentieri A; Doño R ; Macucho M E ; Restuccia A ; Soria H. Patrones de consumo de bebidas y estado dentario en adolescentes Bol. Asoc.Odont. Para Niños (2008) 37(1) 25-28

14. Nestlé M. Wing R. Birch L, Di Sogra L, Drewnowski A. Behavioral and social influences on food choice. Nut Rev (1998); 56 :S50- 74

15. Argentieri A,Pistochini A, Doño R, Añón MC, Tognaccioli L.Determinaciones Analíticas de bebidas no alcohólicas. Bol. Asoc.Odont.Para Niños (2002), 31(4) 09-14

16. Millward A, Shaw L, Smith AJ, Rippin JW, Harrington E. The distribution and severity of tooth wear and the relationship between erosion and dietary constituents in a group of children. Int J Paediatr Dent. (1994) Sep;4(3):151-7.

17. Deshpande S, Hugar S Dental erosion in children : an increasing clinical problem J Indian Soc Ped Prev Dent,(2004) 22(3) $118-127$

Recibido: 15 -10- 2010

Envío revisión: 16 -10- 2010

Aceptado: 22 -11- 2010

Correspondencia: apistoch@hotmail.com 